Sir,

\section{Peripheral idiopathic polypoidal choroidal vasculopathy}

Idiopathic polypoidal choroidal vasculopathy (IPCV) is also known as posterior uveal bleeding syndrome, or multiple recurrent serosanguineous retinal pigment epithelial detachment syndrome. IPCV is characterised by a vascular abnormality that originates from the inner choroid and leads to episodic, serosanguineous or haemorrhagic detachments of retinal pigment epithelium and retina. ${ }^{1-5}$ The vascular abnormality is composed of a network of branching vessels of variable dimensions that terminate in aneurysm-like enlargements. Clinically these enlargements appear as reddish-orange, spheroidal or polyp-like structures on fundus examination. ${ }^{4}$

Although most previous reports have emphasised the typical peripapillary location of the vascular lesion, a less common macular variant was reported by Moorthy et al. ${ }^{5}$ in 1998. In this report, we present a case of peripheral IPCV, which is extremely rare. To date, only one such case has been published, and two other cases have been mentioned in the literature. ${ }^{6}$

\section{Case report}

A 65-year-old white man was presented with a complaint of decreased vision in his right eye for 2 months. His best corrected visual acuity was counting fingers in his right eye, and 20/20 in his left eye. The anterior segments and intraocular pressures were normal in both eyes. Fundus examination of the right eye revealed a serosanguineous retinal detachment involving the lower quadrant and macula, an area of subretinal haemorrhage in the lower temporal quadrant, and reddish-orange polyp-like structures at the posterior border of the subretinal haemorrhage site (Fig. 1). The left fundus was normal.

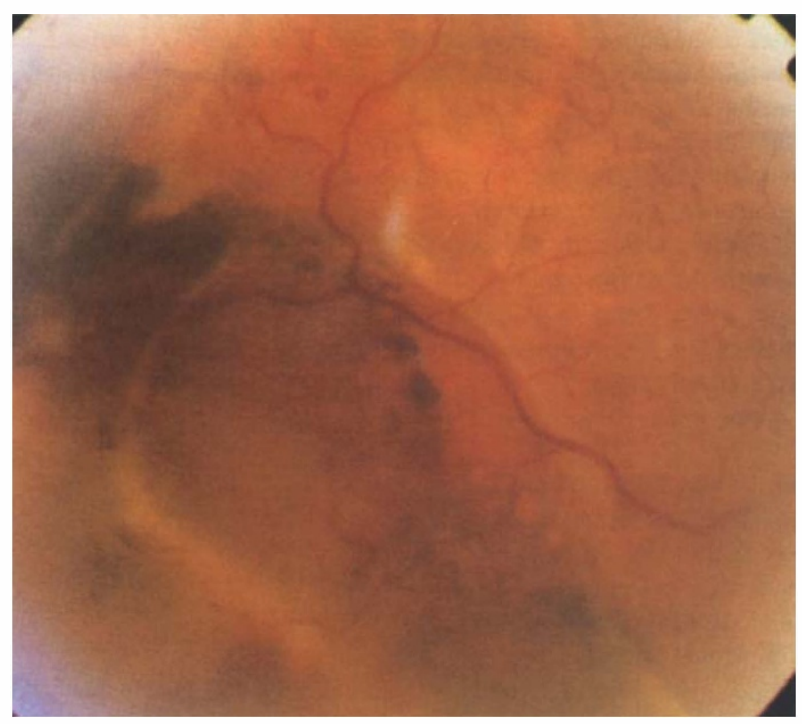

Fig. 1. Fundus photograph showing retinal detachment and subretinal haemorrhage in the lower temporal quadrant of the right eye.
Fundus fluorescein angiography showed patchy areas of staining and leakage in the lower temporal quadrant and blockage of choroidal fluorescence by blood in the area of subretinal haemorrhage. Indocyanine green (ICG) angiography revealed multiple aneurysm-like dilatations of choroidal vessels in the area corresponding to the subretinal haemorrhage site seen on fundus examination of the lower temporal quadrant (Fig. 2a). Staining and leakage from these lesions were observed in the later stages of ICG angiography (Fig. 2b). Fundus fluorescein and ICG angiography of the left eye were normal.

Therapy for the right eye involved laser photocoagulation of the hyperfluorescent lesions identified on ICG angiography. During follow-up, the exudation and retinal elevation diminished. At his last examination, the patient's final visual acuity in the right eye had improved to counting fingers at $2 \mathrm{~m}$.

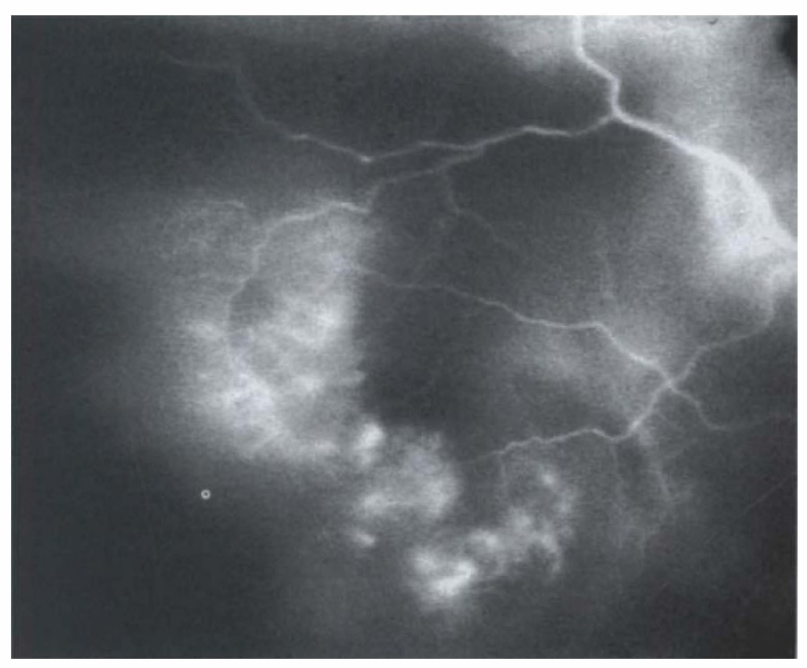

(a)

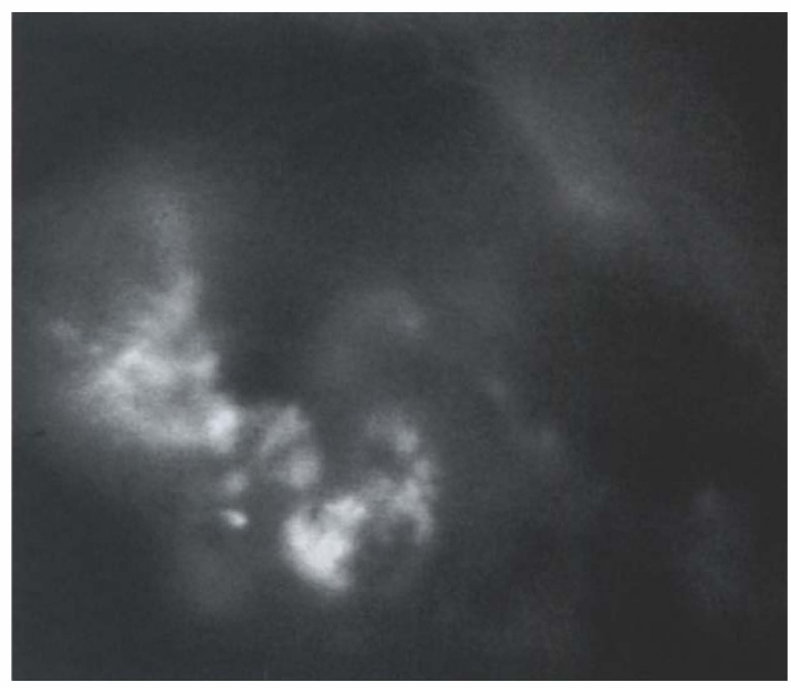

(b)

Fig. 2. (a) Mid-phase indocyanine green angiogram of the lower temporal quadrant of the right eye showing multiple aneurysm-like dilatations of choroidal vessels. (b) Late phase indocyanine green angiogram of the lower temporal quadrant of the right eye showing staining and leakage from the aneurysm-like dilatations. 


\section{Comment}

IPCV is a vascular disorder that originates from the choroidal circulation. The incidence of the condition is highest in black women, but IPCV occurs in both sexes and in other races as well. ${ }^{4}$ The characteristic vascular lesions are usually found in a peripapillary location, but there are a growing number of reports on macular IPCV cases that do not have peripapillary involvement. ${ }^{1-5}$ In a recent article, $94 \%$ of the lesions were macular in Japanese patients. ${ }^{7}$ Although the number of peripapillary and macular IPCV cases is rising, peripheral IPCV is still very rare. The reasons for the rarity of this form, and for the differences between races with regard to location of IPCV lesions, are unknown.

ICG angiography is essential in the diagnosis of IPCV, and for guidance in focal laser photocoagulation. ${ }^{4,5}$ The best way to manage patients with IPCV remains unclear, but laser photocoagulation of the polypoidal choroidal lesions is advised if there is subretinal fluid accumulation, if hard exudates are present, or if haemorrhage is threatening the fovea. ${ }^{5}$ The benefits of laser photocoagulation have already been described. ${ }^{5}$ In our case, laser photocoagulation was done because of the patient's low visual acuity and the fact that the detachment involved the macula. However, the patient's visual acuity improved only slightly during follow-up. This may have been due to the long-standing detachment of the fovea. The use of a diode laser for treating IPCV has been suggested in one report, but experience with this modality is limited at present. ${ }^{8}$

IPCV must be differentiated from various other ocular pathologies because of its unique course and the treatment required. With regard to differential diagnosis, subretinal choroidal neovascularisation secondary to age-related macular degeneration remains the most important pathology. Although both conditions are vascular and originate in the choroid, the lack of drusen and focal hyperpigmentation, the reddish-orange colour of the lesions on fundus examination, and the sharply demarcated, nodular pattern of the lesions on ICG angiography differentiate IPCV from subretinal choroidal neovascularisation secondary to age-related macular degeneration. Inflammatory and neoplastic disorders of the choroid must also be included in differential diagnosis. ${ }^{9}$

In the presented case we made the diagnosis of IPCV on the basis of the typical appearance of the lesions on fundus examination and ICG angiography. The rarity of the peripheral location makes this IPCV case interesting. In the future, performing ICG angiography on patients who have no history of trauma but who exhibit subretinal haemorrhage at the periphery of the fundus, may reveal more cases of peripheral IPCV.

\section{References}

1. Yanuzzi LA, Sorensen JA, Spaide RF, Lipson B. Idiopathic polypoidal choroidal vasculopathy (IPCV). Retina 1990;10:1-8.
2. Stern RM, Zakov N, Zeggara H, Gutman FA. Multiple recurrent serosanguineous retinal pigment epithelial detachments in black women. Am J Ophthalmol 1985;100:5560-9.

3. Kleiner RC, Brucker AJ, Johnston RL. The posterior uveal bleeding syndrome. Retina 1990;10:9-17.

4. Yanuzzi LA, Ciardella A, Spaide RF, Rabb M, Freund B, Orlock DA. The expanding clinical spectrum of idiopathic polypoidal choroidal vasculopathy. Arch Ophthalmol 1997;115:478-85.

5. Moorthy RS, Lyon AT, Rabb MF, Spaide RF, Yanuzzi LA, Jampol LM. Idiopathic polypoidal choroidal vasculopathy of the macula. Ophthalmology 1998;105:1380-5.

6. Yanuzzi LA, Nogueira FB, Spaide RF, Guyer DR, Orlock DA, Colombero D, Freund KB. Idiopathic polypoidal choroidal vasculopathy: a peripheral lesion. Arch Ophthalmol 1998;116:382-3.

7. Uyama M, Matsubara T, Fukushima I, Matsunaga H, Iwashita K, Nagai Y, Takabashi K. Idiopathic polypoidal choroidal vasculopathy in Japanese patients. Arch Ophthalmol 1999;117:1035-42.

8. Gomez-Ulla F, Gonzalez F, Torreiro MG. Diode laser photocoagulation in idiopathic polypoidal choroidal vasculopathy. Retina 1998;18:481-3.

9. Spaide RF. ICG videoangiography of idiopathic polypoidal choroidal vasculopathy. In: Yanuzzi LA, Flower RW, Slakter JS, editors. Indocyanine green angiography. St Louis: Mosby, 1997:329-40.

Ahmet Akman ${ }^{1}$

Haluk Akbatur ${ }^{2}$

Gürsel Yilmaz ${ }^{1}$

Pinar Aydin ${ }^{1}$

'Department of Ophthalmology

Baskent University

Faculty of Medicine

Ankara, Turkey

${ }^{2}$ Private Practice

Ankara, Turkey

Ahmet Akman, MD

Göz Hastaliklari ABD

Baskent Üniversitesi Hastanesi

10.Sokak

06490 Bahcelievler

Ankara, Turkey

Tel: +90 3122150349

Fax: +9031222373 33

e-mail: choroid@excite.com

Sir,

Idiopathic sclerochoroidal calcification

Idiopathic sclerochoroidal calcification (ISC) is a rare benign disorder which may be confused with the usually solitary lesions of choroidal osteoma that are most often found in young females 10-30 years of age. ${ }^{1,2}$ In contrast, ISC generally occurs in older patients (mean age 76 years), with multiple lesions which are typically outside the foveal region, usually leaving visual acuity unaffected. ISC is characteristically bilateral. ${ }^{1,3}$ Other entities which may be confused with ISC are choroidal metastases, amelanotic melanoma and choroidal granuloma. Choroidal metastatic carcinoma lesions are generally larger, thicker, more well defined and often associated with serous retinal detachment. 